\title{
Nanofiltration Hollow Fiber Membranes Made from Sulfonated Polysulfone having a Cyanophenylene Group
}

\author{
Masao Higashi, Takahito Nakao, Junsuke Morita and Tooru Kitagawa*
}

\author{
Membrane-Structural Development Group, Toyobo Co., Ltd, Research Center, 2-1-1 Katata, Otsu, Shiga \\ 520-0292, Japan
}

\begin{abstract}
A nanofiltration hollow fiber membrane made from sulfonated polysulfone was proposed in this work to meet the demands of having tolerance against chemicals. The sulfonate group in the molecule is a source of highly hydrophilic properties and may increase the inter-molecular force acting between molecules on which it is attached. It also contributes to forming a tight structure in the membrane. The membrane may produce higher water flux than those of commercially available nanofiltration membranes made from polyamides. The state of water in the wet membrane was examined with a nuclear magnetic resonance spectrometer. The bonding force to confine water molecules in the membrane may be considered to control the water flux and salt rejection of membranes. It is revealed that there were two kinds of water in the membrane and the salt rejection was raised when the interaction to the water molecules from sulfonate groups in the sulfonated polysulfone molecule was increased. The salt rejection and water flux is highly correlated with the chemical shift of constrained water.
\end{abstract}

Keywords: Nanofiltration, Hollow fiber membrane, sulfonated polysulfone, salt rejection, nuclear magnetic resonance spectroscopy.

\section{INTRODUCTION}

Sulfonated polysulfone is a polymer that can be used to make nanofiltration membranes. The molecule has two different parts which are hydrophobic and hydrophilic [1, 2]. It is well known that the sulfonated polysulfone exhibits relatively higher chemical tolerance than other materials that are used to synthesize nanofiltration membranes such as polyamides [3]. The sulfonate group is a source of highly hydrophilic properties and may increase the inter-molecular force acting between molecules on which it is attached. It also contributes to forming a tight in water. To overcome these disadvantages we put a cyanophenylene group in the molecular structure of sulfonated polysulfone, shown in Figure 1 [4]. This would induce strong inter-molecular interactions to make dense and network structures of sulfonated polysulfone in the membrane down to the nanoscale level. Also, it gives a hand for membranes to keep their own shape without dissolving in water.

Because flat-sheet membranes had a limitation to utilize their area for filtration, we, therefore, tried to make a hollow fiber membrane using sulfonated polysulfone. This adoption is because we can make

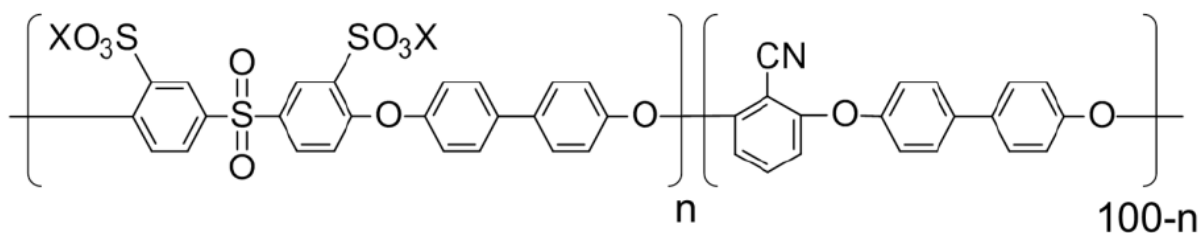

Figure 1: Chemical structure of sulfonated polysulfone $(X=N a$ and $n=23)$ having a cyanophenylene group.

structure in the membrane, the network of which prevents small molecules dissolved in water from passing through the selecting layer of it. As one may imagine, the high content of sulfonate groups in the molecule, going over a certain level, may give higher flux of water, but it may also form a very soft membrane that cannot keep even its own shape as membranes and the resultant structure would dissolve

*Address correspondence to this author at the Membrane-Structural Development Group, Toyobo Co., Ltd, Research Center, 2-1-1 Katata, Otsu, Shiga 520-0292, Japan; E-mail: Tooru_Kitagawa@toyobo.jp use of the advantage of the configuration of hollow fiber membranes that exhibit greater membrane surface area in a unit volume when they are used as a module.

\section{EXPERIMENTAL}

\subsection{Making the Membrane}

A hollow fiber membrane was made using a dry-jet wet spinning method. First, a concentrated solution was prepared from sulfonated polysulfone and 1methyl-2-pyrrolidone (NMP). The range of 
concentration was adjusted from 30 to 40 weight percent. The method for synthesis and polymerization of the polymer is shown in the literature [4]. To make an inner hole of the hollow fiber, a liquid was injected into the center of the concentrated solution that was also extruded in a spinneret with a pump. The nozzle for extrusion had a configuration of a double-cylinder structure. The hollow fiber extruded was led to a coagulation bath, then to a washing process. It was followed by collecting fibers using a wind-up drum. To raise the ability of salt rejection, the membrane was soaked in a bath for annealing. The liquid filled in it was water or $4.5 \%$ salt water at $90{ }^{\circ} \mathrm{C}$ and the duration time for annealing was $20 \mathrm{~min}$.

\subsection{Measuring Flux and Salt Rejection}

Water flux and salt rejection were estimated by keeping hollow fiber membranes in a sealed tube filled with water containing sodium chlorine under a certain temperature and pressure. Both ends of the membrane were cut open and stuck out of the tube; filtered water came out when pressures were applied to the liquid in the tube [5]. Operating conditions of the test were as follows; the temperature was $25{ }^{\circ} \mathrm{C}$, the applied pressure was $1 \mathrm{MPa}$, and the concentration of sodium chloride solution was $1,500 \mathrm{ppm}$. The water filtered with hollow fiber membranes for a certain period of time was weighed on a balance to estimate flux. The salt concentration of the filtered water was estimated from electric conductivity; then the salt rejection was estimated by calculating the following equation:

Salt rejection $=\left(1-C_{A} / C_{B}\right) \times 100[\%]$

where, $\mathrm{C}_{\mathrm{A}}$ stands for the salt concentration of filtered water and $C_{B}$ for the concentration of salt water in the tube.

\subsection{Nuclear Magnetic Resonance Measurement}

A chemical shift for the proton of water molecules in hollow fiber membranes was measured by proton nuclear magnetic resonance (NMR) spectroscopy. First, a hollow fiber membrane was cut, as the length of samples was maintained $5 \mathrm{~cm}$, and was soaked in pure water for longer than $10 \mathrm{~min}$. The membrane was washed with a large amount of pure water $(\mathrm{pH}=7)$ to remove and wash residual solvents and impurities. After taking out of the hollow fiber membrane from the water bath, it was inserted into a capillary tube with a diameter of $3 \mathrm{~mm}$, then the tube having the wet membrane was inserted into an NMR tube with a diameter of $5 \mathrm{~mm}$. The NMR tube was set to an Avance 500 spectrometer of Bruker Co., Ltd (USA). The operating condition of the measurement was as follows; the measurement frequency was $500 \mathrm{MHz}$, the spin rate was $13 \mathrm{~Hz}$, the solvent for sim-lock was deuterated water, the cumulated number was 16 , the repetition time was $5 \mathrm{sec}$., and the temperature was 30 ${ }^{\circ} \mathrm{C}$. The value of chemical shift of free water at the peak-top position was set to $5.24 \mathrm{ppm}$ as a standard.

\section{RESULTS AND DISCUSSION}

First, we look at the relationship between salt rejection of $\mathrm{NaCl}$ (sodium chloride), flux of filtered water and polymer concentration in making hollow fiber membranes with a dry-jet wet spinning method. The concentration of polymer solution and the salt content of coagulant were changed. The results are shown in Figure 2. It is seen that the salt rejection correlated with polymer concentration and the coagulation with a solution of $14.5 \% \mathrm{NaCl}$ also contributed to the raise of salt rejection. There is less correlation between water flux and polymer concentration.

It is important to point out that the state of water in the membrane would be assigned according to the degree of interactions of the water molecules bound to sulfonate groups in the polymer chain, because the spatial distribution of sulfonate groups in the membrane was heterogeneous and the water would be bound to sulfonate groups if it stays in the vicinity of them. We may see different bonding states of water according to the distance of bound water from them. Usually the sulfonate group forms aggregated particles and their difference would also contribute to the bound state of water. The knowledge about the state of water leads us to invent a noble idea to get more efficient membranes having high water flux together with high salt rejection. A proton nuclear resonance spectrum was taken to elucidate the bound state of water. It was used to detect the degree of chemical constraint and motion of hydrogen atoms of water from the polymer chain including sulfonate groups in the membrane. Also some portion of water may be trapped in a small space of tight structures physically in the membrane. They would all influence the motion of water that comes through a membrane wall in filtering pure water from salt water. Figure 3 shows a typical proton magnetic resonance spectrum. The measurement was carried out by keeping the sample hollow fiber membranes fully wet with pure water. We can confirm two peaks in the spectrum; one was assigned as free water and the other was constrained by sulfonate groups. 


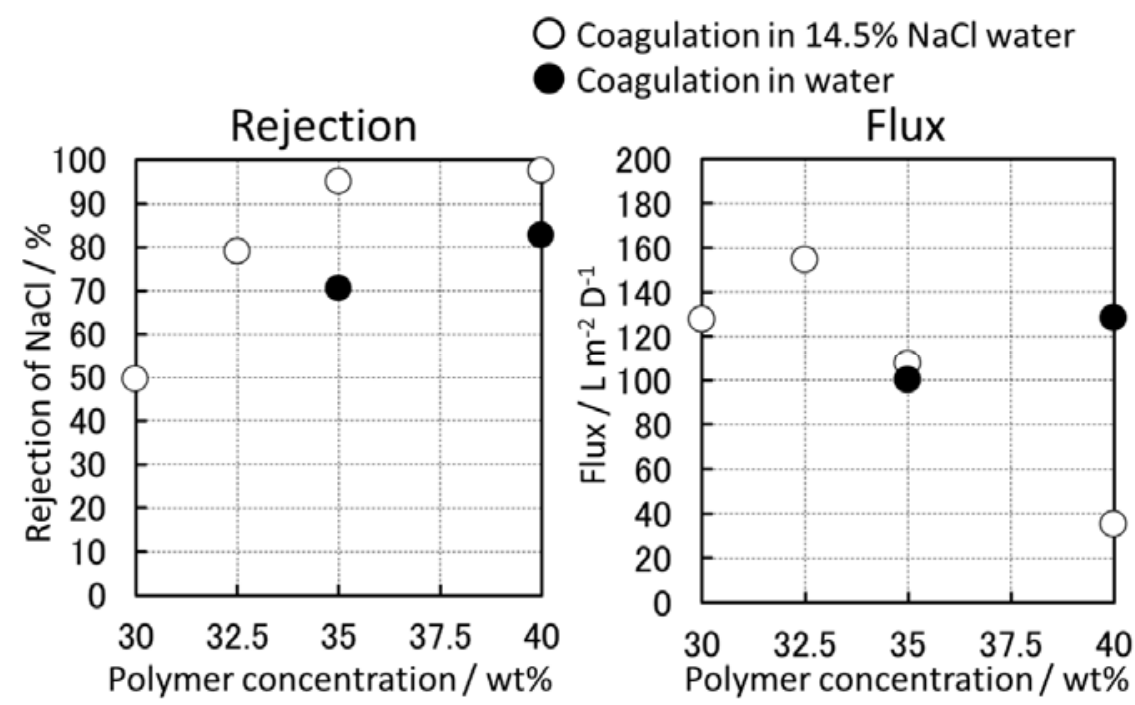

Figure 2: Comparison between rejection of $\mathrm{NaCl}$, flux and polymer concentration when the hollow fiber membrane was made. No annealing was applied as post-treatment.

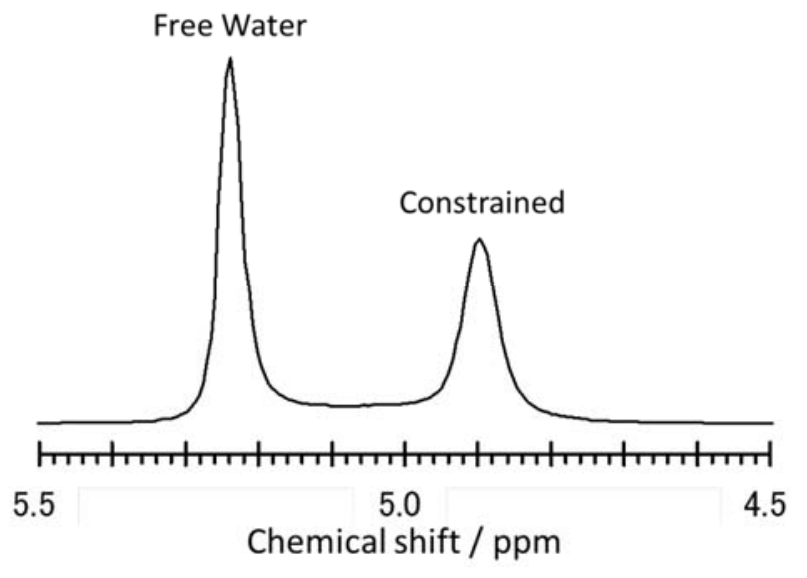

Figure 3: Typical proton magnetic resonance spectrum for protons of water in the membrane.

There were at least two states of water contained in the membrane. Here, the difference between the two peaks in chemical shift would depend on the degree of interactions of bound water with sulfonate groups. Also, the limitation of motion for water molecules would be related to the width of the peaks. According to the chemical shift, it is considered that the peak at 5.24 ppm was due to free water that has no chemical interactions with polymer chains and the peak at 4.90 ppm was related to constrained water chemically by sulfonate groups. It should be also noted that the peak at $4.90 \mathrm{ppm}$ showed enough narrow width close to that of the free water at $5.24 \mathrm{ppm}$, indicating that the constrained water still behaved like free water in motion even it was controlled under the chemical constraint of sulfonate groups. The difference in the chemical shift from free water may be related to the ability of rejecting the passage of salt through membranes and the resemblance in the behavior of free water may contribute to the easiness of permeation for water molecules through membranes.

Figure 4 shows the relationship between salt rejection, water flux and $\Delta$ chemical shift. Here, the $\Delta$ chemical shift is defined as the distance between the chemical shift value of constrained water and that of free water. If water molecules are set under the influence of sulfonate groups, the environment of the proton of water is different than that of free water, so the measured chemical shift value is also different. The data plotted in the figure contain values of membranes made with different conditions; it is seen that the resultant salt rejection and water flux was well explained as a function of $\Delta$ chemical shift. As one can see the bigger the $\Delta$ chemical shift, the higher the salt rejection and the lower the water flux. This $\Delta$ chemical shift was related to the degree of constraint affecting between the proton of water molecules and the sulfonate group in the membrane.

The existence of two kinds of water in the membrane indicates the coexistence of free water that is released from the influence of chemical interactions and constrained water that is attached to the hydrophilic part in the molecule. The value of $\Delta$ chemical shift for the constrained water increases as the flux decreases, indicating the decrease of constraint of water molecules from the sulfonate group in the membrane gives high flux. Here, we can confirm a tendency that higher constraint for water molecules from the sulfonate group gives more ability to separate salt ions by electric repulsion forces and tight structures of membranes with high rejection. 

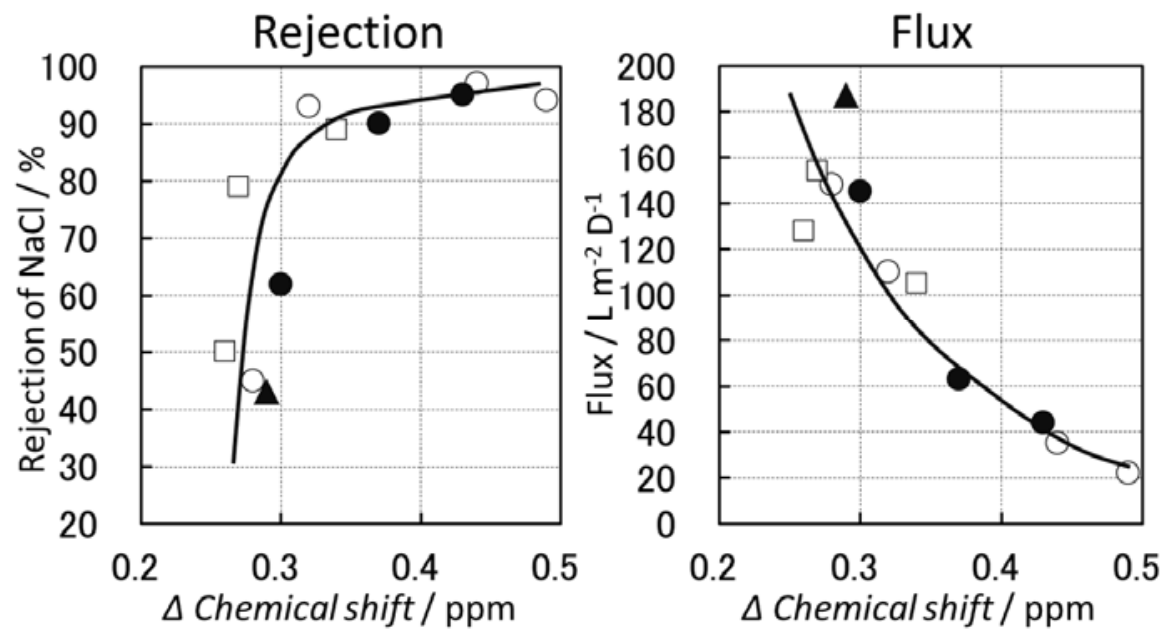

Figure 4: Relationship between salt rejection, flux and $\Delta$ chemical shift. 0 : coagulated in $14.5 \% \mathrm{NaCl}$ water at $25{ }^{\circ} \mathrm{C}$ and annealed in $4.5 \% \mathrm{NaCl}$ water at $90{ }^{\circ} \mathrm{C}$, $\square$ : coagulated in $14.5 \% \mathrm{NaCl}$ water at $25{ }^{\circ} \mathrm{C}$ and no annealing, $\bullet$ : coagulated in water at $25{ }^{\circ} \mathrm{C}$ and annealed in $4.5 \% \mathrm{NaCl}$ water at $90^{\circ} \mathrm{C}, \boldsymbol{\Delta}$ : coagulated in water at $25^{\circ} \mathrm{C}$ and annealed in water at $90{ }^{\circ} \mathrm{C}$.

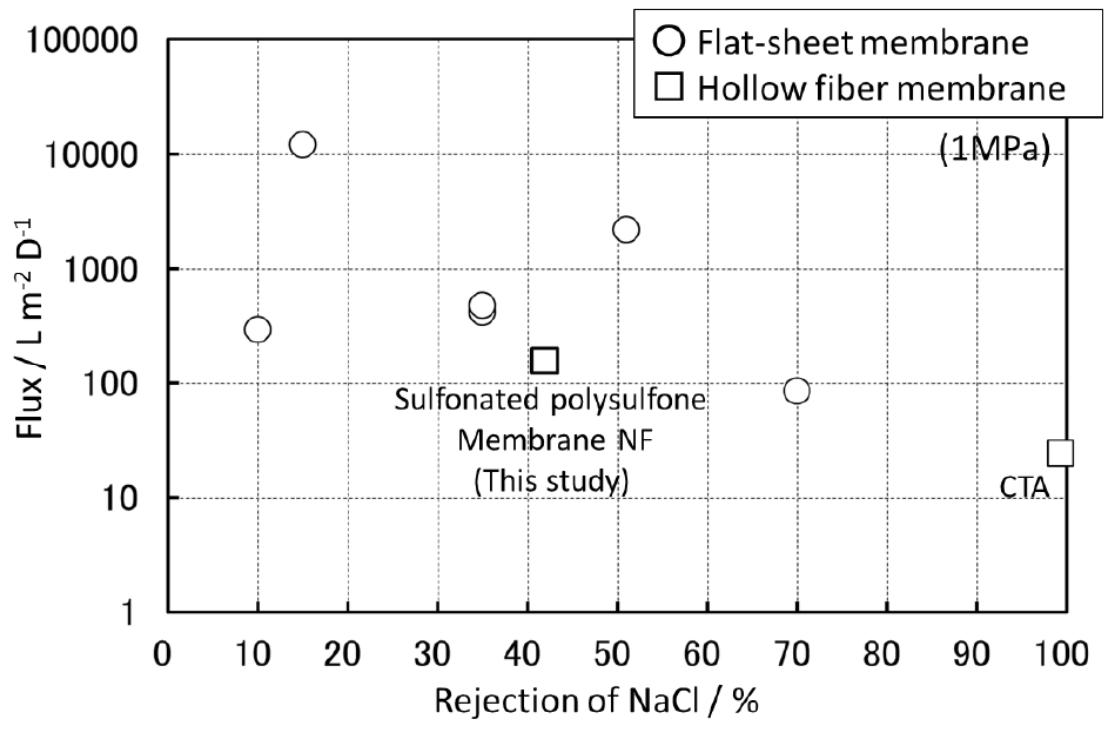

Figure 5: Comparison between a sulfonated polysulfone membrane proposed in this study and other commercial membranes. Here, the values of flux plotted are adjusted at a pressure of $1 \mathrm{MPa}$ because they are measured under different pressures [1,5]. NF stands for nanofiltration and CTA for cellulose triacetate.

The properties of the membrane proposed in this study are compared with other commercially available membranes in Figure 5 [5]. Water flux is plotted against salt rejection. It should be noted that all the commercial membranes plotted in the figure are flat-sheet type except for Toyobo's reverse-osmosis membrane made from cellulose triacetate (CTA). Even though the nanofiltration membrane proposed in this study is a hollow fiber type, its flux is close to the level of commercially available membranes in the area having the same salt rejection. We believe that this tendency is very promising because it would suggest a possibility of realizing a module that would produce more amount of water in a unit volume of it with hollow fiber nanofiltration membranes made from sulfonated polysulfone proposed in this study.

It is also found that there were at least two states of water in the membrane. But we don't have enough information to explain how water molecules were separated from the mixture of salt and water from the microscopic point of view. Therefore, it is necessary for us to investigate into the structure of membrane, especially to make clear how the sulfonate group aggregates into particles and separate water molecules from salt water. The way of connection between the particles may be related to the efficiency of separating water molecules from salt water [6], because it would lead water molecules from the salt water with rejecting 
the passage of salt through membranes. The structure of particles should be made through phase separation. Because this membrane proposed in this study has a certain level of resistance against chemicals including the acid and base, it would be applicable to a forward osmosis system; some draw solutions used for the system show basic.

\section{CONCLUSIONS}

A sulfonated polysulfone having a cyanophenylene group in its molecule could improve the hollow fiber membrane properties for nanofiltration application. The flux of membrane proposed in this study was in the level of other commercially available nanofiltration membranes. The state of water in the membrane is analyzed with proton nuclear magnetic resonance spectroscopy, indicating that there was water constrained by sulfonate groups. The salt rejection and water flux highly correlated with the chemical shift of constrained water.

\section{ACKNOWLEDGEMENTS}

Measurements of NMR were performed by the help of Mr. Yamane at Analytical Research Center in Toyobo Co., Ltd, for which the authors express their gratitude. Thanks are also extended to Dr. Sekino of ex-cooperate officer of Toyobo Co., Ltd. for his valuable comments.

\section{REFERENCES}

[1] Ikeda K, Nakano T, Ito H, Kubota T, Yamamoto S. New composite charged reverse osmosis membrane. Desalination 1988; 68: 109-119.

http://dx.doi.org/10.1016/0011-9164(88)80048-1

[2] Koyama K, Nishimura M. Preparation and performance of sulfonated polysulfone charged membrane for reverse osmosis. Membrane 1980; 5: 189-193. http://dx.doi.org/10.5360/membrane.5.189

[3] Park HB, Freeman BD, Zhang ZB, Sankir M, McGrath JE. Highly chlorine-tolerant polymer for desalinations. Angew Chem 2008; 120: 6108-6113.

http://dx.doi.org/10.1002/ange.200800454

[4] Sakaguchi Y, Kitamura K, Yamashita M, Takase S, Takasugi $\mathrm{K}$, Akitomo $\mathrm{Y}$. Synthesis and properties of sulfonated poly(arylene ether)s with flexible oligometric phenylene ether segments. Macromolecules 2012; 45: 5403-5409. http://dx.doi.org/10.1021/ma300665x

[5] Schlesinger R, Gotzinger G, Sixta H, Friedl A, Harasek M. Evaluation of alkali resistant nanofiltration membranes for the separation of hemicellulose from concentrated alkaline process liquors. Desalination 2006; 192: 303-314. http://dx.doi.org/10.1016/j.desal.2005.05.031

[6] Kim YS, Dong L, Hickner MA, Glass TE, Webb V, McGrath JE. State of water in disulfonated poly(arylene ether sulfone) copolymers and a perfluorosulfonic acid copolymer (nafion) and its effect on physical and electrochemical properties. Macromolecules 2003; 36: 6281-6285.

http://dx.doi.org/10.1021/ma0301451

(C) 2016 Higashi et al.; Licensee Lifescience Global.

This is an open access article licensed under the terms of the Creative Commons Attribution Non-Commercial License (http://creativecommons.org/licenses/by-nc/3.0/) which permits unrestricted, non-commercial use, distribution and reproduction in any medium, provided the work is properly cited. 